Digital Press Social Sciences and Humanities

La politesse de la requête des locuteurs natifs du français : quelles stratégies, quelles modifications?

Elvi Syahrin and Ratna

Proceeding of Conférence internationale sur le français 2018

Joesana Tjahjani, Merry Andriani, Sajarwa, Wening Udasmoro (eds) 


\title{
La politesse de la requête des locuteurs natifs du français : quelles stratégies, quelles modifications?
}

\author{
Elvi Syahrin ${ }^{*}$ et Ratna ${ }^{2}$ \\ 1Universitas Negeri Medan, Sumatera Utara, Indonesia \\ 2Universitas Negeri Jakarta, Jakarta, Indonesia \\ *e-mail : elsyachrian@yahoo.com
}

\section{Résumé}

La politesse est spécifique à la culture et sensible au contexte. Pour réussir une interaction, il faut savoir « ce qui est habituel ou attendu dans une situation donnée au sein d'une communauté. En effet, les cadres dépendent uniquement des cultures et des communautés dans lesquelles ils sont créés » (Terkourafi, 2005). Voilà pourquoi ce qui est poli dans une communauté peut être considéré comme impoli dans une autre et, bien évidemment, ce qui est poli dans un contexte particulier pourrait être impoli dans un autre. Le présent article offre une étude pragmatique particulièrement sur la politesse de l'acte de langage de requête. Cette étude porte sur les stratégies de politesse de la requête réalisées par les locuteurs natifs du français. Participée par 10 locuteurs natifs du français comme répondants, elle examine la politesse selon le schéma de codage élaboré dans le cadre du Cross-Cultural Speech Act Realization Project (CCSARP) (Blum-Kulka, House, \& Kasper, 1989) modifiée par Warga (2005). En utilisant le Discourse Completion Test (DCT) écrit pour la collecte de données, la recherche met l'accent sur les modificateurs internes de la requête adaptée de Bae (2012). Il se compose de 6 situations de conversations sélectionnées de Reiter (2000). Les variables sociales correspondantes à 3 systèmes de politesse de Scollon \& Scollon (2001) est également devenu la base de l'analyse dans cette étude. Les résultats de notre recherche ont démontré que les locuteurs natifs du français ont tendance à réaliser leurs requêtes en stratégies indirectes conventionnelles par l'utilisation de la phrase interrogative. La sous-stratégie utilisée est Question sur une Condition de Réussite (QCR) dominée par la question sur la capacité. Alors que la plupart des modificateurs internes utilisés pour montrer la politesse est l'utilisation du mode conditionnel. Ces résultats pourraient avoir des implications pour les apprenants du FLE, les enseignants, les auteurs de manuels, les concepteurs de syllabus et, les chercheurs.

\section{Mots-clés}

politesse, requête, stratégies, modifications, Français

\begin{abstract}
Politeness is culture-specific and context sensitive. Usually, speakers of a language community acquire strategies of politeness from their ongoing discussions with each other in daily life. That is why what is polite in a community can be considered rude in another and what is polite in a particular context could be rude in another. This article offers a particularly pragmatic study on the politeness of the speech acts of query. This study examines the politeness of the request strategies carried out by French native speakers. Participated by 10 native speakers as referees, it examines the politeness according to the encoding scheme developed in Cross-Cultural Speech Act Realization Project (CCSARP) (Blum-Kulka, House, \& Kasper, 1989) modified by Warga (2005). Using Discourse Completion Test (DCT) for data collection, this research, at the same time, focuses on the internal change of request adapted by Jin Ah (2012). It consists of 6 situations selected conversations of Reiter (2000). Social variables corresponding to 3 systems of politeness of Scollon and Scollon (2001) also became the basis of the analysis in this study. The results of our research have shown that French native speakers tend to make their request for conventional indirect strategy by the use of the interrogative sentence. The sub-strategy which was used is question on a condition of success dominated by the question on the ability. While most of the internal changes to show politeness is the use of the conditional mode. These findings could have implications for
\end{abstract}


the learners of French as foreign language, teachers, authors of textbooks, syllabus designers and researchers.

\section{Keywords}

politeness, query, strategies, changes, French

\section{Introduction}

Le phénomène de la politesse linguistique fait partie de la recherche linguistique en Europe occidentale et en Amérique du Nord depuis plus de trente ans et se situe généralement dans le domaine de la pragmatique et de la sociolinguistique (Watts, 2003, p. 53).

La politesse est un cas fondamental dans la pragmatique parce que la politesse est un phénomène universel dans l'utilisation de la langue dans un contexte social (Brown et Levinson, 1987). En général, la politesse se définie comme la propriété sociale, qui est une action dans laquelle une personne fait preuve d'un comportement régulier en respectant les autres conformément aux normes fondues dans la société.

L'étude de la politesse a été liée à la théorie de l'acte de langage (Austin 1962 ; Searle 1969) et aux notions de "face" positive et négative de Brown et Levinson $(1978$; 1987). Parmi les divers actes de langage, l'acte de requête joue un rôle important reflétant la politesse dans l'interaction entre les personnes. Il est considéré comme un bon indicateur de la manière dont les personnes de cultures différentes s'expriment et interagissent (Blum-Kulka, 1991 ; Sifianou, 1992). En particulier, la requête peut refléter la politesse d'une langue parce que c'est un Face Threatening Acts (FTA) qui exige des stratégies de politesse pour diminuer "l'imposition des énoncés".

La politesse est liée à l'indirection. Reiter (2000 : 41) déclare que la politesse et l'indirection ont toujours été liées. Cela conforme à la proposition de Brown et Levinson (1978) et Leech (1983), qui perçoivent que l'indirection dans la requête s'emploi principalement comme un moyen de transmettre la politesse.

Le lien entre l'indirection et la politesse est supporté loin avant par l'observation de Searle (1975 : 76 ) qui affirme que la politesse est la motivation la plus importante pour l'indirection de requête, et certaines formes tendent à devenir les manières conventionnellement polies de faire des requêtes indirectes. Exemple :

(1) « Voudrais-tu m'aider à faire cet exposé ? ».

(2) «Aide-moi à faire cet exposé ».

(Blum-Kulka et al., 1989).

La requête (1) est considérablement plus polie que celle de (2).

L'énoncé (1) est sous une forme interrogative qui donne le choix à l'interlocuteur d'exécuter ou de ne pas exécuter la requête. Il est donc considéré comme plus poli. Ce type de stratégie est appelé la stratégie indirecte conventionnelle (Blum-Kulka et al. 1989).

Searle (1975) souligne également qu'il existe deux types de l'indirection : les stratégies indirectes conventionnelles et les stratégies indirectes non-conventionnelles. Les premières se réfèrent aux actes conçus qui sont exécutés à des fins fonctionnelles qui ne sont pas limitées par leurs formes grammaticales telles que :

(3) «Pourrais-tu me passer le sel ?»

Tandis que les indirects non-conventionnels consistent en des énoncés plus ambigus. Par exemple, l'énoncé comme ci-après peut être considéré comme un indice pour demander à l'interlocuteur de fermer la fenêtre.

(4) «As-tu froid ?»

A côte de l'utilisation de l'indirection, il existe d'autres moyens d'augmenter le degré de politesse, à savoir les modificateurs pragmatiques (Leech, 2014). En utilisant des termes différents ; les atténuateurs, Blum-Kulka et al. (1989) et Reiter (2000) suggèrent que les atténuateurs, atténuent les effets négatifs des actes de langage qui pourraient menacer la face négative de locuteur. La présence de dispositifs tels que 
des modificateurs syntaxiques et lexicaux, internes ou externes, et des mouvements de soutien peut atténuer l'impact de la force de la requête. Par exemple, l'énoncé (5) est supposé être plus poli que (6).

(5) « Montre-moi le couloir, s'il te plait».

(6) « Montre-moi le couloir».

La politesse est spécifique à la culture et sensible au contexte. Cela signifie que de nombreuses cultures ont leurs propres moyens de montrer la politesse, la déférence, l'honneur et la reconnaissance du statut social des interactants. Les normes de politesse propres à la culture se développent dans le comportement de communication des différentes communautés de discours. En outre, la politesse est un phénomène plus sensible au contexte et socialement diversifié. Par exemple, les normes de politesse sociale qui se rapportent à un dîner en famille sont assez différentes de celles concernant un dîner officiel.

Pour être socialement accepté et maintenir une communication harmonieuse avec les autres, un locuteur doit avoir des stratégies. Lorsqu'il s'agit de choisir des stratégies de politesse, plusieurs facteurs peuvent influencer le choix. Selon Brown \& Levinson (1987 :15), le choix du degré de politesse dépend du degré de gravité (Rate of imposition) du FTA, la distance sociale (Distance) qui existe entre les interlocuteurs, de leur relation de pouvoir (Power). Premièrement, la distance sociale (aussi appelée intimité entre interlocuteurs) est basée sur le degré de connaissance ou de fréquence des interactions entre les interlocuteurs.

En lien avec ces stratégies, Scollon \& Scollon (2001) observent trois systèmes de politesse : le système de politesse de Déférence, le système de politesse de Solidarité et le système de politesse de Hiérarchie. La distinction des trois systèmes repose principalement sur l'existence d'une différence de pouvoir et sur la distance sociale entre les interlocuteurs.

Le système de politesse de Déférence est un système dans lequel les participants sont considérés égaux, ou presque égaux, mais se traitent à distance (par exemple, camarades de classe). Dans un système de politesse de Solidarité, les locuteurs ne ressentent pas qu'il y a de différence de pouvoir entre eux (par exemple, les amis). Le système de politesse de Hiérarchie peut être reconnu par les organisations éducatives, les entreprises, les gouvernements et les locuteurs utilisant des stratégies de politesse différentes.

Habituellement, les locuteurs d'une communauté linguistique acquièrent des stratégies de politesse à partir de leurs échanges continus les uns avec les autres dans les quotidiens. Voilà pourquoi ce qui est poli dans une communauté peut être considéré comme impoli dans une autre et, parallèlement, ce qui est poli dans un contexte particulier pourrait être impoli dans un autre.

Dans l'apprentissage des langues étrangères, la compétence pragmatique est considérée comme l'aspect de la langue le plus difficile à maîtriser (Blum Kulka \& Sheffer, 1993, p. 219). Un apprenant d'une langue étrangère peut être capable de prononcer des mots clairement ou être capable d'utiliser correctement des phrases complexes et longues, mais sans être accompagné d'une compétence pragmatique, il n'est pas garantie qu'il puisse communiquer socialement propre avec certaine fluidité.

Malheureusement, la compréhension de la politesse est souvent moins perçue dans beaucoup de classes de FLE. La plupart des apprenants se concentrent excessivement sur le développement de la compétence grammaticale en français que sur le développement de la compétence pragmatique. On n'est pas assez sensible qu'être un apprenant d'une langue étrangère signifie apprendre tous les aspects de la langue cible, y compris le système de politesse utilisé par les locuteurs natifs.

Cela va avec Frame-based view de Terkourafi (2005:99) qui affirme que les cadres fournissent la base d'une approche véritablement universalisant. Pour réussir une interaction, il faut savoir « ce qui est habituel ou attendu dans une situation donnée au sein d'une communauté. En effet, les cadres dépendent uniquement des cultures et des communautés dans lesquelles ils sont créés » (Terkourafi, 2005, p. 110). Ainsi, pour chaque situation, il existe un cadre différent dans lequel un certain type de comportement est poli.

Cet article discute la réalisation de la politesse de l'acte de requête des locuteurs natifs du français selon certaine situation et certain système de politesse. La discussion se porte, effectivement, sur les stratégies utilisées par les locuteurs natifs du français, à savoir le degré l'indirection et les modificateurs internes.

\section{Méthode}

Les données de la recherche sont obtenues par la distribution des questionnaires écrits en forme de DCT (Discourse Completion Test) remplis par mél. Les sujets de cette recherche sont 10 locuteurs natifs du 
français qui résident à Metz, Lyon, Marseille, la Rochelle, Saint Aunès, Caen, Bayonne, et Lorraine. Le questionnaire se contient de 6 d'actes de requête selon l'instrument de Reiter (2000) qui, originellement, sont 12 situations de l'acte de requête en Anglais. Ces situations sont choisies parmi 12 situations conformément à 3 variables sociales proposées par Brown \& Levinson (1987) et le système de politesse de Scollon \& Scollon (2001).

Les données obtenues sont réécrites en tenant compte des variables sociales basées sur la théorie du système de politesse de Scollon \& Scollon (2001) tirée de la théorie de Brown \& Levinson (1987) qui divise : P comme le pouvoir, $\mathrm{D}$ comme la distance sociale et $\mathrm{R}$ comme la gravité de l'imposition. Dans cette étude, les actes de langage de requête sont classés de manière similaire à diverses variables sociales (Brown et Levinson, 1987), qui est également utilisées par Reiter (2000).

L'instrument de recherche a été compilé basé sur le système de politesse de Scollon \& Scollon (2001), qui est divisé en trois principaux types de systèmes de politesse qui peuvent être observés dans divers contextes. Ce système de politesse est basé sur la différence de Pouvoir (+ P ou -P) et la Distance sociale entre les participants (+ D ou -D). Ce système s'appelle un système de politesse de Déférence (formel), un système de politesse de Solidarité (informel) et un système de politesse de Hiérarchie.

Le tableau suivant présente une cartographie des instruments de recherche sous la forme d'une classification de la DCT (Data Collection Test) basée sur des variables sociales dans chaque système de politesse de Scollon \& Scollon (2001).

Tableu 1 Le système de politesse Scollon \& Scollon (2001)

\begin{tabular}{|l|l|c|c|c|}
\hline \multicolumn{1}{|c|}{$\begin{array}{c}\text { Systèmes de } \\
\text { politesse }\end{array}$} & \multicolumn{3}{|c|}{ Variables sociales } \\
\hline & & $\begin{array}{c}\text { Pouvoir } \\
\text { (P) }\end{array}$ & $\begin{array}{c}\text { La distance } \\
\text { sociale } \\
\text { (D) }\end{array}$ & $\begin{array}{c}\text { Degré de } \\
\text { gravité } \\
\text { (R) }\end{array}$ \\
\hline Déférence & & $(-\mathrm{P})$ & $(+\mathrm{D})$ & Faible/fort \\
\hline Solidarité & & $(-\mathrm{P})$ & $(-\mathrm{D})$ & Faible/fort \\
\hline Hiérarchie & & $(+\mathrm{P})$ & $(+\mathrm{D})$ & Faible/fort \\
\cline { 2 - 5 } & & $(+\mathrm{P})$ & $(-\mathrm{D})$ & \\
\hline
\end{tabular}

\section{Résultats et discussions}

\subsection{La réalisation de la politesse de l'acte de requête des locuteurs natifs du français selon le degré d'indirection}

Le classement de la politesse d'actes de langage de requête comprend des situations tirées de 12 situations formulées par Reiter (2000), ce sont :

1. Changer la place dans le bus,

2. Emprunter la voiture d'un voisin,

3. Demander une direction,

4. Emprunter une maison pour les vacances,

5. Emprunter des livres à son professeur,

6. Emprunter une voiture à sa supérieure.

Tableu 2 La réalisation de la politesse de l'acte de requête selon le degré d'indirection

\begin{tabular}{|l|l|c|c|}
\hline \multicolumn{1}{|c|}{ Stratégies } & \multicolumn{1}{|c|}{ Sous-Stratégies } & Fréquence & $\begin{array}{c}\text { Pourcentage } \\
\%\end{array}$ \\
\hline I. Formulation Direct & 1. Mode dérivable & 5 & 8.3 \\
\cline { 2 - 4 } & 2. Performatifs & - & - \\
\cline { 2 - 4 } & 3. Performatifs délimités & - & - \\
\cline { 2 - 4 } & 4. Obligation & - & - \\
\cline { 2 - 4 } & $\begin{array}{l}\text { 5. Affirmation d'un désir ou } \\
\text { d'une nécessité }\end{array}$ & - & 1.6 \\
\hline
\end{tabular}




\begin{tabular}{|c|c|c|c|}
\hline \multirow[t]{7}{*}{ II. Formulation Indirect } & \multirow{7}{*}{$\begin{array}{l}\text { 6. Formule Suggestive } \\
\text { 7. Préparation/Question sur la } \\
\text { Condition de Réussite (QCR): } \\
\text { a. Question sur la capacité } \\
\text { b. Question sur la possibilité } \\
\text { c. Question sur la volonté } \\
\text { d. Question sur la disponibilité } \\
\text { e. Question sur la permission } \\
\text { 8. Consultative }\end{array}$} & 1 & 1.6 \\
\hline & & & \\
\hline & & $\frac{21}{13}$ & $\begin{array}{c}35 \\
21.6\end{array}$ \\
\hline & & - & - \\
\hline & & 8 & 13.3 \\
\hline & & - & - \\
\hline & & 8 & 13.33 \\
\hline \multirow[t]{2}{*}{$\begin{array}{l}\text { III. Formulation Indirect non- } \\
\text { conventionnels }\end{array}$} & 9. Allusion & 3 & 5 \\
\hline & & 60 & 100 \\
\hline
\end{tabular}

En général, les locuteurs natifs ont tendance à réaliser leur requête en utilisant la stratégie indirecte (Formulation Indirect) pour montrer la politesse des actes de langage de requête par l'utilisation des phrases interrogatives. L'utilisation de ce type de stratégie comporte la politesse de haut niveau. Ceci est conforme à l'avis de Leech (1983), qui affirme que plus un énoncé est indirect, plus l'énoncé devient poli.

Par rapport à la sous-stratégie utilisée, la plupart des locuteurs natifs utilisent la sous-stratégie de Question sur la Condition de Réussite (QCR) de la question sur la capacité, qui consiste à utiliser une phrase interrogeant la capacité du partenaire en utilisant le verbe "pouvoir", par exemple :

(7) «Pourriez-vous le prêter?»

Ainsi, la forme de la phrase interrogative utilisant le verbe « pouvoir » est généralement utilisée.

Une autre sous-stratégie souvent utilisée est l'utilisation de la stratégie de Question sur la Condition de Réussite (QCR) sur la question sur la possibilité. C'est une phrase qui pose la question sur la possibilité d'accomplir la requête, par exemple :

(8) «Est-il possible d'occuper la dernière semaine?»

Enfin, Les locuteurs natifs utilisent également Allusion pour masquer la requête soumise en utilisant des phrases fermées telles que

(9) «Je n'ai pas de voiture ... ».

La requête implicitement est transmise par la phrase affirmative.

\subsection{La réalisation de la politesse de l'acte de requête des locuteurs natifs du français selon l'utilisation de modificateurs internes}

Le tableu 3 présente l'utilisation de modificateurs internes dans 6 situations.

Tableu 3 La réalisation de la politesse de l'acte de requête selon l'utilisation des modificateurs internes

\begin{tabular}{|l|l|l|l|l|l|l|l|l|l|l|l|l|l|c|}
\hline $\begin{array}{l}\text { Situa- } \\
\text { tion }\end{array}$ & $\begin{array}{l}\text { Systeme } \\
\text { Politesse }\end{array}$ & $\begin{array}{l}\mathbf{N} \\
\mathbf{e} \\
\text { g. }\end{array}$ & $\begin{array}{l}\text { Md } \\
\text { Pol } \\
\text {. }\end{array}$ & $\begin{array}{l}\text { Co } \\
\text { nd } \\
\text {. }\end{array}$ & $\begin{array}{l}\text { DM } \\
\text { od/ } \\
\text { t. }\end{array}$ & $\begin{array}{l}\text { M } \\
\text { in }\end{array}$ & $\begin{array}{l}\text { Do } \\
\text { wn } \\
\text {. }\end{array}$ & $\begin{array}{l}\text { MC } \\
\text { ons } \\
\text {. }\end{array}$ & $\begin{array}{l}\text { Su } \\
\text { bj. }\end{array}$ & $\begin{array}{l}\text { Ed } \\
\text { Ag. }\end{array}$ & $\begin{array}{l}\text { Ap } \\
\text { pr. }\end{array}$ & $\begin{array}{l}\text { T } \\
\text { e } \\
\text { n. }\end{array}$ & $\begin{array}{l}\text { Idte } \\
\text { mps }\end{array}$ & $\begin{array}{l}\text { To } \\
\text { tal }\end{array}$ \\
\hline $\mathbf{1}$ & Déférence & 0 & 2 & 5 & 0 & 0 & 0 & 3 & 0 & 2 & 0 & 0 & 0 & 12 \\
\hline $\mathbf{2}$ & Déférence & 2 & 2 & 3 & 0 & 0 & 0 & 2 & 0 & 1 & 0 & 1 & 0 & 11 \\
\hline $\mathbf{3}$ & Solidarité & 0 & 2 & 0 & 0 & 0 & 0 & 0 & 0 & 0 & 0 & 0 & 1 & 3 \\
\hline $\mathbf{4}$ & Solidarité & 1 & 0 & 1 & 0 & 0 & 0 & 4 & 0 & 1 & 0 & 2 & 0 & 9 \\
\hline $\mathbf{5}$ & Hiérarchie & 1 & 1 & 5 & 0 & 5 & 0 & 2 & 0 & 3 & 0 & 0 & 0 & 17 \\
\hline $\mathbf{6}$ & Hiérarchie & 1 & 1 & 3 & 0 & 1 & 0 & 2 & 0 & 3 & 0 & 2 & 0 & 13 \\
\hline \multicolumn{2}{|l|}{} & 5 & 8 & 17 & 0 & 6 & 0 & 13 & 0 & 10 & 0 & 5 & 1 & 65 \\
\hline
\end{tabular}


Note :

1) Neg. = Négation

2) MdPol. = Marquer de politesse

3) Cond. = Conditionnel

4) $\mathrm{DMod} / \mathrm{t}=$ Désactualisateur modal ou temporal

5) Min. = Minimisateur

6) Down. = Downtoner

7) MCons. = Mesure Consultative

8) Subj. = Subjectivisation

9) EdAg. = Évitement de l'agent

10) Appr. = Appréciation

11) Tent. $=$ Tentative

12) IdTemps = Intensificateur du temps (Upgrader)

Le tableau 3 nous montre que le modificateur interne le plus souvent utilisé pour démontrer la politesse est l'utilisation du mode conditionnel, dans la phrase :

(10) « Pourrais-tu m'aider à faire avec la tienne ?»

Cela montre que le conditionnel joue un rôle important pour montrer la politesse d'un énoncé.

L'autre modificateur souvent utilisé est la Mesure Consultative, c'est la proposition d'options à l'interlocuteur afin qu'il puisse coopérer avec le locuteur. Le locuteur utilise la formulation rituelle telle que :

\section{(11) « Ça ne vous dérange pas de changer la place avec moi ?»}

L'utilisation de cette formulation montre le niveau de la relation informel et de politesse dans lequel le locuteur veut se montrer proche de leur interlocuteur, de sorte que leur interlocuteur ne se sent pas mal à l'aise et ne se sent pas opposé aux demandes du locuteur.

On a conclu qu'il y a plusieurs systèmes de politesse utilisés dans l'acte de requête analysée :

1. Dans une situation de requête avec un système de politesse de Déférence (formel) : $(-P /+D / R$ est faible), c'est quand la variable sociale de pouvoir (P) entre le locuteur et l'interlocuteur est petit (-), la distance est loin (+), et le degré de gravité est faible, donc la requête peut être réalisée par:

a. L'utilisation de la stratégie de politesse indirecte (formulation indirecte) à travers une phrase interrogative en mettant l'accent sur :

(1) Question sur la disponibilité :

(12) «Auriez-vous la gentillesse de prendre la place libre qui est à coté ?»

(2) Consultative:

(13) « Ça vous dérangerait de changer de place avec moi ?»

(3) Question sur la possibilité :

(14) «Serait-il possible que nous intervertissions nos places?»

(4) Question sur la capacité :

(15) «Vous pourriez vous décaler?»

b. Le choix de modificateurs internes de :

(1) Conditionnel

(2) Mesure consultative

(3) Marquer de politesse

(4) Evitement de l'agent

2. Dans une situation de requête avec un système de politesse de Solidarité (informel) : (-P/+D/R est fort), c'est quand la variable sociale de pouvoir (P) entre le locuteur et l'interlocuteur est petit (-), la distance est loin (+), et le degré de gravité est fort, donc la requête peut être réalisée aussi par :

a. L'utilisation de la stratégie de politesse indirecte (formulation indirecte) à travers une phrase interrogative en mettant l'accent sur :

(1) Question sur la possibilité : 
(16) « Ce serait possible que je l'occupe la dernière semaine?»

(2) Question sur la capacité :

(17) «Tu penses que tu pourrais me la prêter?»

(3) Consultative :

(18) «Est-ce que tu accepterais de me louer ta maison de campagne pendant une semaine?»

(4) Formule suggestive :

(19) «Et si tu me prêtais ta maison pendant une semaine?»

b. Formulation indirecte non-conventionnelle- Allusion :

(20) «Si jamais elle est *dispo, je le suis aussi. »

c. Le choix de modificateurs internes de :

(1) Mesure consultative

(2) Tentative

(3) Conditionnel

(4) Négation

(5) Evitement de l'agent

3. Dans une situation de requête avec un système de politesse de Hiérarchie $(+P /+D / R$ est faible $)$, c'est quand la variable sociale de pouvoir (P) entre le locuteur et l'interlocuteur est grand $(+)$, la distance est loin $(+)$, et le degré de gravité est faible, donc la requête peut être réalisée aussi par :

a. L'utilisation de la stratégie de politesse indirecte (formulation indirecte) à travers une phrase interrogative en mettant l'accent sur :

(1) Question sur la capacité :

(21) «Pourriez-vous me le prêter?»

(2) Question sur la possibilité :

(22) « Est-ce que ça serait possible d'aller à votre bureau pour vous l'emprunter?»

(3) Question sur la disponibilité :

(23) «Auriez-vous l'amabilité de me prêter le livre?»

(4) Consultative :

(24) «Est-ce que ça ne vous dérangerait pas de me le prêter quelques jours?»

b. Le choix de modificateurs internes de:
(1) Conditionnel
(2) Minimisateur
(3) Evitement de l'agent
(4) Mesure consultative
(5) Négation
(6) Marquer de politesse

Cette recherche nous a permis ainsi de constater que les locuteurs natifs français, comme la plupart des locuteurs natifs de langues romanes ont tendance à utiliser la formulation indirecte comme une des stratégies de politesse préférées dans les situations formelles, informelles et hiérarchie. La formulation indirecte réduit les obligations des deux locuteurs en interaction et les décharge ainsi de la responsabilité directe en donnant les choix à l'interlocuteur.

L'utilisation de la stratégie indirecte pourra donner la contribution positive pour les apprenants du FLE Indonésiens puisqu'on exprime très fréquemment ce type de stratégie en requête mais comme la politesse dépends beaucoup au contexte, il y a des possibilités que les apprenants, influencé par leur culture d'origine, aient la difficulté de choisir quelles stratégies utilisées et quelles modifications appliquées pour telles situations. Donc, Il est important que les apprenants soient sensible à la dynamisation des cultures cibles en profitant les contacts, le plus fréquents possible, avec des locuteurs natifs.

Les résultats de la recherche pourraient également avoir des implications sur les didactiques du FLE, notamment pour les apprenants, les enseignants, les auteurs de manuels, les concepteurs de syllabus et les futurs chercheurs.

En tout cas, il y a encore de place suffisant pour explorer le domaine de politesse, ainsi que celui de l'impolitesse. Nous pourrons, par exemple, reprendre et élargir l'intérêt principal de notre projet original sur l'étude d'Interlangue, c'est à dire, de comparer la politesse de locuteurs natifs et non natifs sur d'autres actes de langages potentiellement menaçant ; critiquer, interroger, interdire, etc. 


\section{Conclusion}

Basé sur les données obtenues, on peut conclure que la politesse de l'acte de langage de requête est réalisée par la stratégie indirecte (Formulation indirecte) avec l'utilisation de la phrase interrogative. L'emploie de ce type de stratégie comporte la politesse de haut niveau. Ceci est conforme à l'avis Leech (1983) qui suggère que l'indirection rend la politesse ; le plus indirect que l'on exprime, le plus poli serait notre discours. Les sous-stratégies couramment utilisées sont Question sur la Condition de Réussite (QCR) en présentant (1) Question sur la capacité, c'est l'utilisation de la phrase interrogative qui demande la capacité de l'interlocuteur en utilisant le verbe « pouvoir », (2) Question sur la possibilité ; l'utilisation de la phrase interrogative qui demande la possibilité de l'interlocuteur. Il se trouve également l'utilisation de (3) Allusion ; utilisation d'une stratégie visant à masquer les demandes soumises à l'aide de phrases fermées délivrées par le type de phrase affirmative.

La politesse de l'acte de langage de requête est réalisée également par la présence de Modificateurs internes du mode Conditionnel. Cela nous montre que dans ce contexte, le mode Conditionnel joue un rôle important pour montrer la politesse dans un discours. En outre, une forme de Mesure Consultative a également été trouvée, cela fait par proposer les choix aux interlocuteurs pour qu'ils coopèrent avec les locuteurs. L'utilisation de ce type de modificateurs internes montre le degré de l'intimité et la politesse informel.

\section{Références}

Austin, J. L. (1962). Quand dire, c'est faire. Paris: Éditions du Seuil.

Bae, J. A. (2014). La politesse dans la communication interculturelle : les stratégies de politesse utilisées par les coréens apprenant le français comme langue seconde lors des situations de demande. Université de Montréal, Montréal.

Blum-Kulka, S. (1991). nterlanguage pragmatics: The case of requests. In R. Phillipson, E. Kellerman, L.

Selinker, M. Sharwood Smith, \& M. Swain (Eds.), Foreign/second language pedagogy research (pp. 255272). Clevedon : Multilingual Matters.

Blum-Kulka, S., \& Sheffer, H. (1993). The Metapragmatic Discourse of American Israeli Families at Dinner. In G. Kasper \& S. Blum-Kulka (Eds.), Interlanguage Pragmatics (pp. 196-223). Oxford: Oxford University Press.

Blum-Kulka, S., House, J., \& Kasper, G. (1989). Cross-Cultural Pragmatics: Requests and Apologies. Noorwood : Ablex. https://doi.org/10.2307/415556

Brown, P., \& Levinson, S. (1978). Universals in language usage: Politeness phenomena. In Questions and politeness: Strategies in social interaction (pp. 56-311). Cambridge: Cambridge University Press.

Brown, P., \& Levinson, S. (1987). Politeness: Some Universals in Language Usage. Cambridge: Cambridge University Press. https://doi.org/10.2307/3587263

Koller, A., \& Searle, J. R. (2006). Speech Acts: An Essay in the Philosophy of Language. Language. https://doi.org/10.2307/412428

Leech, G. (2014). The Pragmatics of Politeness. The Pragmatics of Politeness. Oxford: Oxford University Press. https://doi.org/10.1093/acprof:oso/9780195341386.001.0001

Leech, G. N. (1983). Principles of pragmatics. New York: Long. https://doi.org/10.1017/S0022226700010367

Reiter, R. M. (2000). Linguistic Politeness in Britain and Uruguay: A contrastive study of requests and apologies. Philadelphia: John Benjamins Publishing. 
Scollon, R., \& Wong., S. S. (2001). Discourse and Intercultural Communication. In D. Schiffrin, D. Tannen, \& H. H. E. (Eds.), The Handbook of Discourse Analysis (pp. 538-547). Oxford: Blackwell.

Searle, J. R. (1976). A Classification of Illocutionary Acts. Language in Society, 5(1), 1-23. Retrieved from http://www.jstor.org/stable/4166848

Searle, J. (1969). Speech Acts : An Essay in Philosophy of Language. Cambridge : Cambridge University Press.

Sifianou, M. (1992). Politeness Phenomena in England and Greece : A Cross-Cultural Perspective. Oxford : Oxford University Press.

Terkourafi, M. (2005). An argument for a frame-based approach to politeness (pp. 99-116). https://doi.org/10.1075/pbns.139.10ter

Warga, M. (2005). «Est-ce que tu pourrais m'aider? » vs. « Je voudrais te demander si tu pourrais m'aider » Les requêtes en français natif et en interlangue. Vox Romanica, 64, 141-159.

Watts, R. (2003). Politeness. Cambridge : Cambridge University Press. 